\title{
Magnetic imaging in the presence of external fields: Technique and applications (invited)
}

\author{
Romel D. Gomez \\ Department of Electrical Engineering, University of Maryland, College Park, Maryland 20742 and \\ Laboratory for Physics Sciences, 8050 Greenmead Drive, College Park, Maryland 20740 \\ Edward R. Burke \\ Laboratory for Physics Sciences, 8050 Greenmead Drive, College Park, Maryland 20740 \\ Isaak D. Mayergoyz \\ Department of Electrical Engineering, University of Maryland, College Park, Maryland 20742
}

\begin{abstract}
Magnetic force microscopy (MFM) in the presence of an external magnetic field has been developed. This has led to further understanding of image formation in MFM as well as new insights concerning the interaction of magnetic recording media with an external field. Our results confirm that, at low applied fields, image formation results from the interaction of the component by the local surface field along the direction of the probe's magnetization. By reorienting the probe's magnetization by an appropriate application of an external field, it is possible to selectively image specific components of the local field. At higher applied fields, the probe becomes saturated and the changes in the images may be attributed to magnetization reversal of the sample. We have observed the transformations that occur at various stages of the dc erasure of thin-film recording media. This technique has also been applied to conventional magneto-optical media to study domain collapse caused by increasing temperature with an external bias field. The methods, results, and their analysis are presented. (C) 1996 American Institute of Physics. [S0021-8979(96)57008-5]
\end{abstract}

\section{INTRODUCTION}

Since the first reports of its development in $1987,{ }^{1}$ magnetic force microscopy (MFM) has emerged as a powerful tool in understanding the micromagnetics of surfaces. ${ }^{2}$ Its capability to resolve $\$ 100 \mathrm{~nm}$ features, combined with lenient requirements on sample preparation and operating environments, has made it a workhorse in technology development as well as fundamental investigations of surface ${ }^{3}$ and biological ${ }^{4}$ magnetism. Despite its widespread use, however, most MFM images were obtained under ambient magnetic field conditions. As a result, studies have been limited to samples being either in a locally nonmagnetized or remanent states.

Recently, the capability of imaging samples in the presence of an external magnetic field has been developed. ${ }^{5}$ This has allowed direct imaging of the microscopic evolution of thin-film recording patterns undergoing dc erasure; and has opened up the possibility of investigating microscopic magnetic properties of surfaces at any point along the magnetization curve. In this paper, we provide the details of our technique and discuss the implications of subjecting both the probe and sample to an external field. We focused our studies on thin-film recording and magneto-optical media, not only because of their importance in the storage industry but because they represent excellent case models for in-plane and out-of-plane magnetization structures.

\section{TECHNIQUE}

\section{A. Background}

Magnetic force microscopy generates a two dimensional mapping of the local force-gradient between a magnetic probe and a sample, separated by about a hundred nanom- eters. The probe is comprised of a cantilever beam with a sharp, magnetic tip at one end that is forced to oscillate at its resonance frequency. It is then rastered across the surface while the changes in the oscillation phase of the cantilever are recorded in an array as a function of its two dimensional lateral position. The change of the oscillation phase is directly proportional to the force gradient, so that the resulting array, by using computer graphics, forms an image of the distribution of the local force gradient.

The technique can be understood by considering the motion of the cantilever probe as a single-point mass $m$ undergoing one-dimensional forced harmonic oscillation along the vertical $z$ axis. ${ }^{6}$ The resonance frequency is determined by the intrinsic elastic constant of the cantilever, in combination with a small perturbation due to the probe-surface interaction force $F(z)$. The latter is incorporated by expanding $F(z)$ in a Taylor series and keeping the first order term $F^{\prime}=\partial F / \partial z_{0}$. When the driving frequency is near the resonance, $\omega \sim \omega_{0}$, the local force gradient $F^{\prime}$ will shift the resonance frequency by an amount $\Delta \omega_{0} \approx \omega_{0}\left(F^{\prime} / 2 k\right)$. As a consequence, the phase will vary from its nominal $90^{\circ}$ out-of-phase condition relative to the driving term by an amount

$$
\Delta \phi \approx-2 Q \frac{\Delta \omega}{\omega_{0}}=-\frac{Q F^{\prime}}{k},
$$

where $Q$ is the quality factor of the cantilever. The phase variation at each point is proportional to the local force gradient.

Our commercial device, the Nanoscope III MFM, operates in the so-called lift and phase sensitive detection method. ${ }^{7}$ The topography of the surface is first measured by using atomic force microscopy techniques and the contour is retraced with the probe at a preset height above the surface 


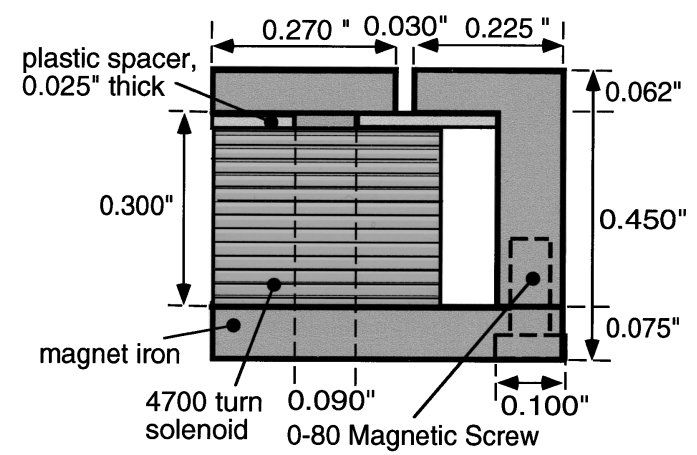

FIG. 1. Electromagnet design for MFM with an external in-plane magnetic field.

as the force-gradient is measured. In this manner, the surface topographical effects are minimized.

Since the probe is coated with a ferromagnetic alloy, the tip-surface force is dominated by the magnetic interaction if short-range adhesion forces ${ }^{8}$ are neglected. For simplicity, we describe the tip as a single constant magnetic dipole, $\mathbf{m}_{\text {tip }}$. The force experienced by this dipole in a local magnetic field $\mathbf{H}$ is ${ }^{2}$

$$
\mathbf{F}=-\left(\mathbf{m}_{\text {tip }} \cdot \boldsymbol{\nabla}\right) \mathbf{H} \text {. }
$$

Only the $z$ component of the force contributes to the oscillation. By differentiating with respect to $z$ and using $\boldsymbol{\nabla} \times \mathbf{H}=0$, Eqs. (1) and (2) yield an explicit expression for contrast formation in phase-detection MFM:

$$
\Delta \phi \approx \frac{Q}{k} \sum_{i} m_{i} \frac{\partial^{2} H_{i}}{\partial z^{2}}
$$

This equation states that as long as the magnetic properties of the sample and the probe are unaffected by their mutual interaction, the contrast in MFM arises from the variations of the second $z$ derivative of the component of the surface field in the direction of the probe's magnetization.

One consequence of Eq. (3) is that it is possible to selectively image specific components of the second derivative of the local surface magnetic field by appropriately orienting the probe magnetization direction. In other words, by a judicious choice of probe magnetization directions, it is feasible to individually map out the $z$ derivatives of the three Cartesian components of the local surface magnetic field vectors. One way, of course, is to prepare probes in a highly controlled manner in order to have the easy axis aligned along definite directions. In principle, specific probes can be used to image particular surface field components. In our experiments, however, the reorientation of the direction of the probe's magnetization is accomplished by applying an external dc magnetic field during imaging. The intention is to orient the probe magnetization by sufficiently low fields which preclude significant effects on the sample.

\section{B. Magnet design}

Adding an external field in MFM involves the incorporation of an electromagnet at the sample stage. Figure 1 shows the critical details of our design which produces mag-

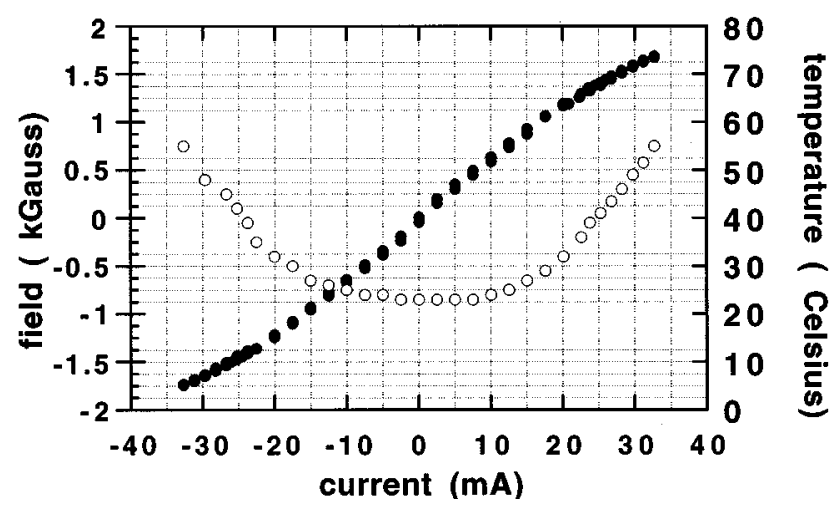

FIG. 2. Magnetic field and temperature calibration vs input current.

netic fields primarily oriented along the sample surface plane. The dimensions were constrained by the available space in our device. The materials and coil specifications were chosen to optimize the magnetic field strength generated at the gap location and subject to a heating constraint to prevent temperature-induced problems, such as drifts, depolarization of the piezoelectric scanners, and heat-induced changes in the sample magnetic properties. The magnetic field generated in the gap of length $L_{g}$ can be estimated as

$$
B_{g} \cong \frac{\mu_{0} N I}{L_{g}} \quad \text { (MKSA units), }
$$

where $N$ is the total number of turns, $I$ is the current, and $\mu_{0}$ is the permeability of free space. This can be derived from Ampere's law for the case where the permeability of the iron core is large. The relevant parameters are: $N=4700$ turns and $L_{g}=0.76 \mathrm{~mm}$. At $30 \mathrm{~mA}, B_{g}=2330 \mathrm{G}$ and it is expected to reach saturation at $B_{g}=4500 \mathrm{G}$ for a current of $56 \mathrm{~mA}$. A measurement of the horizontal component of the field, shown in Fig. 2, was obtained by using a miniature Hall probe (LakeShore HT 2100) which was calibrated against a known field. The data indicate that the magnetic field varies linearly with current from 0 to $30 \mathrm{~mA}$, demonstrating that within this range, the iron yoke is driven at the reversible regime of its magnetization curve. High-frequency fluctuations in the field were found to be about $0.5 \%$. Similarly, the temperature was measured by using a calibrated thermocouple which was spot welded near the gap region. Heating effects, concurrently plotted in Fig. 2, show that a dc current of up to $32 \mathrm{~mA}$ elevates the temperature to less than $55^{\circ} \mathrm{C}$.

\section{RESULTS AND DISCUSSION}

The samples used in our experiment were obtained from conventional and experimental thin film disks as well as conventional magneto-optic samples. The macroscopic properties of some of these samples were previously measured and reported elsewhere. ${ }^{5,9}$

\section{A. Probe effect: Component-resolved imaging}

Confirming the predictions of Eq. (5), the individual components of the local surface magnetic fields of the thin film in-plane recorded medium are shown in Fig. 3. A diagram representing the expected distribution of the magneti- 


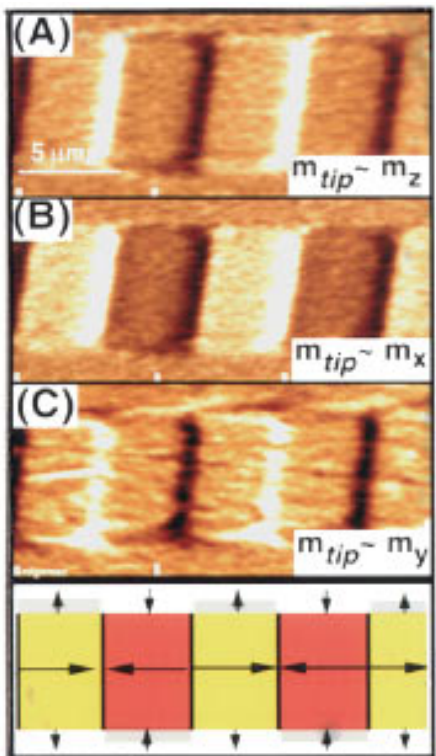

FIG. 3. Component-selective images of a thin-film recording medium. Images obtained with probe nominally oriented along the (A) normal $z$ axis, (B) in-plane $x$ axis, and (C) in-plane $y$ axis, reflecting the contributions of the local surface field at these directions, respectively. Bottom: sketch of the magnetization pattern.

zation distribution is drawn in the bottom of the figure. Figures 3(A) and 3(B) were obtained from the same area of the disk. In Fig. 3(A), the probe was premagnetized by a $3.5 \mathrm{kOe}$ magnet along the vertical direction prior to image acquisition and no external horizontal field was applied to the sample. Neglecting some nonlinear effects due to the probe-sample interaction, the probe magnetization in Fig. 3(A) can be taken as $m \sim m_{z} ; m_{x}, m_{y} \sim 0$. The contrast formation in Fig. 3(A) arises predominantly from $\partial^{2} H_{z} / \partial z^{2}$ and it appears as an alternating series of bright and dark strips that are more or less localized along the transition regions. Note that despite the fact that the pattern is a series of alternating in plane magnetizations, very little contrast can be observed within the interior regions of the patterns. In fact, the image contrast in the interior regions is similar to the background areas outside of the patterns. In Fig. 3(B), which was acquired with a 200 Oe field along the track direction, the interior features have emerged and the two opposite magnetization directions are clearly distinguishable. This arises because the external horizontal dc magnetic field produced a nonvanishing $m_{x}$ component of the probe magnetization which induces a dominant term proportional to $\partial^{2} H_{x} / \partial z^{2}$.

We offer the following qualitative interpretation of these images in terms of the physical quantities such as the magnetic charges and the magnetization. For illustrative purposes, consider the simple case of a one-dimensional sinusoidal magnetization pattern $M_{x}=M_{0} \sin (k x)$, in a medium of thickness $\delta$. The local surface magnetic field components above the surface for this configuration can be computed, ${ }^{10}$ and the corresponding second derivatives with respect to $z$ are

$$
\frac{\partial^{2} H_{z}}{\partial z^{2}} \propto \nabla \cdot M=\frac{\partial M_{x}}{\partial x}
$$

and

$$
\frac{\partial^{2} H_{x}}{\partial z^{2}} \propto M_{x} .
$$

In other words, MFM imaging with the $z$-axis probe orientation yields images that are proportional to the divergence of the magnetization or the volume magnetic charge density while the $x$-axis orientation yields images that are proportional to the distribution of magnetization. In real systems, the images may deviate from this simple interpretation, albeit only slightly. For example, for an in-plane arctangent transition with a transition length of $150 \mathrm{~nm}$, the main difference between the divergence of the magnetization and the second derivative of the field with $z$ is the broadness of the peak. The FWHM widths are $400 \mathrm{~nm}$ for $\partial M / \partial x$ and $200 \mathrm{~nm}$ for $\partial^{2} H_{z} / \partial z^{2} \cdot{ }^{11}$ Once instrumental resolution limits of about 100 $\mathrm{nm}$ are taken into account, the distinction becomes less conspicuous. Naturally, in order to obtain quantitative results, one would have to use Eq. (3) to derive the actual image of magnetization from a set of MFM raw images.

By extending this approach, we have selectively imaged the crosstrack or $y$ component ${ }^{12}$ as shown in Fig. 3(C). This is obtained by preparing the sample such that the tracks are perpendicular to the direction of the applied field. Note that apart from the contrast at the transition regions, pairs of localized bright and dark areas are visible along both edges of the track. These crosstrack features are distinctive in that a predominantly bright edge structure is paired with a dark edge structure on the opposite edge in any specific "bit." (By a "bit," we mean the entire rectangular area having a constant magnetization direction.) These edge features are clearly distinct from the edges in Fig. 3(A), although both are manifestations of the same magnetic feature. In one case, the transverse component of the edge magnetization (as drawn in the diagram) is detected, and on the other, the $z$ component or the magnetic charges accompanying the edge magnetizations are measured. The intensity of the features are relatively weak in comparison with the main transitions, which explains the higher noise contribution in the image. In general, they depend upon the combined effects of the head geometry and a weakly oriented recording medium. ${ }^{12}$

While the images still show partial mixing between the individual components of the field, they nevertheless clearly support the assertions of Eq. (3). The recurring influence of the $z$ contribution may have resulted from the combination of two effects, namely, the external field having a component along the $z$ direction or the probe having a slight anisotropy favoring vertical alignment.

\section{B. Probe hysteretic effects}

Having established the reorientation of the probe due to the external field, we now study the probe hysteretic behavior along the $x$ direction. Specifically, we focus on the transformation of the images as the horizontal field is cycled between moderately large negative and positive values. The 


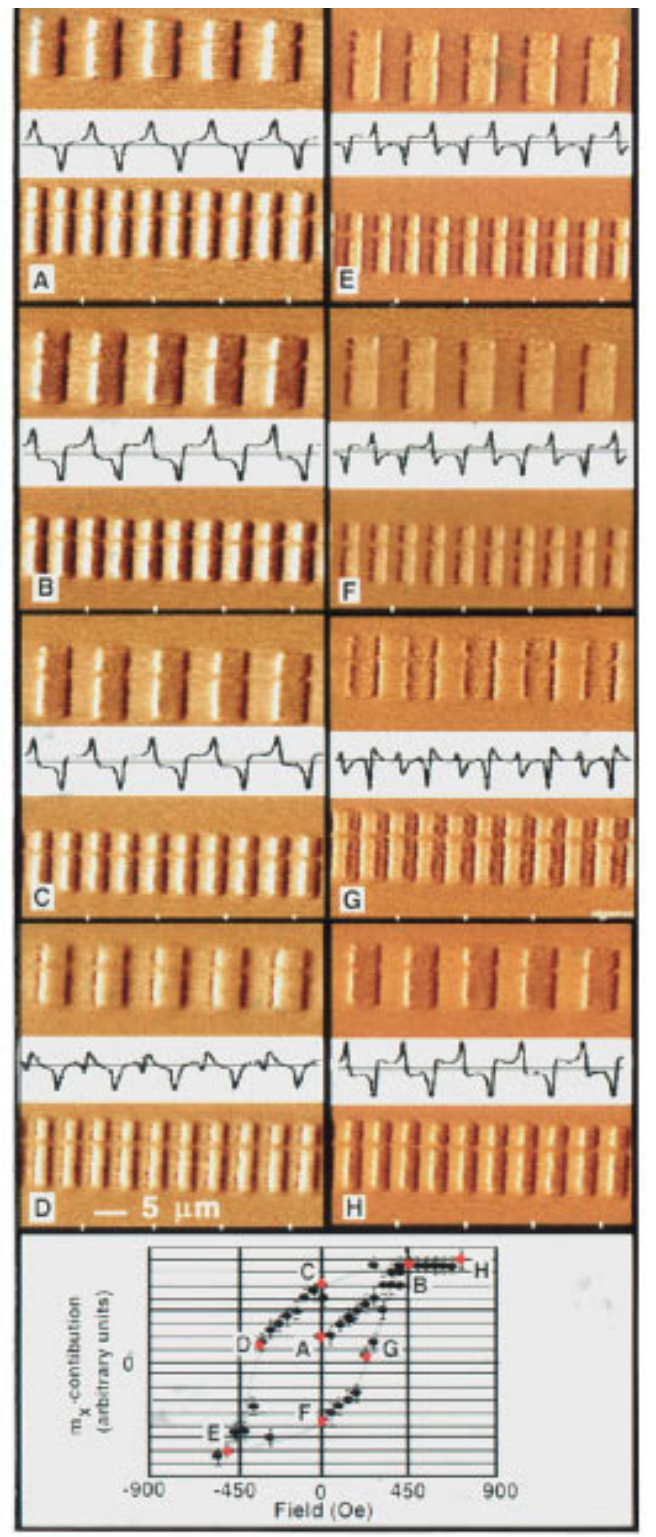

FIG. 4. Hysteretic behavior of MFM probe magnetization along $x$ axis and corresponding images at saturation, remanence, and coercivity.

main goal is to derive, from the images, the changes in the $m_{x}$ component of the probe under an external bias dc field.

The result is summarized in Fig. 4. The sequence was started by premagnetizing the probe along the vertical direction and acquiring the initial image. The horizontal field was slowly raised up to $+500 \mathrm{Oe}$, ramped back down to -500 Oe, and again reversed direction to +700 Oe. Images were acquired at each field increment all along the cyclic path. The plot at the center of the figure is an actual measurement of the probe's horizontal magnetization component. Each data point along this curve was derived by averaging the line profiles of the upper (long wavelength) track as shown in the images. Since the images were obtained from the same area and the external field was low in comparison with the coercivity of the medium $\left(H_{c}>1600 \mathrm{Oe}\right)$, then the magnetization component of the probe along the track direction can be derived from the images and Eq. (3). Ideally, this can be done by fitting each of the experimental lineshapes with the modeled response given by Eq. (3) and extracting the relative coefficients $m_{x}$ and $m_{z}$. However, since the $z$-component contribution is confined at the transition regions, we simply derived the $m_{x}$ contribution for each image by measuring the average contrast levels at the midpoints of the "bits" while keeping track of the separate magnetization directions. The resulting plot clearly exhibits the hysteretic property of the probe.

Special points along the probe's magnetization loop are shown in images $4(\mathrm{~A})-4(\mathrm{H})$. They depict how changing the probe magnetization alters the image formation. The contrast mechanism with no applied field is shown in image 4(A), after performing the standard procedure of pre-magnetizing the probe along the $z$ direction. The features are confined exclusively at the transition regions and thus, the horizontal probe magnetization components $m_{x}$ can be taken to be nearly zero at this initial state. This is a widely used implementation of the MFM and most images of thin-film media in the literature belong in this category.

The contribution of the horizontal component increases linearly with the field until it attains the contrast shown in 4(B). Further increases of the external field do not produce significant variations since the probe's magnetization is saturated along the direction of the field. Once the field has been removed, however, image 4(C) differs only slightly from the saturated state, indicating that horizontally realigned domains maintain their orientation. This large remanent magnetization can be beneficial in being able to premagnetize the probe along the horizontal direction instead of the customary vertical direction, and increase its sensitivity in detecting the local horizontal field contribution. Note, however, that while the horizontal component is large, the contribution from the $z$ component is still quite significant. This implies that the probe contains numerous domains, some of which have strong anisotropy favoring magnetization along the $z$ direction.

The onset of $m_{x}$ reversal is illustrated in 4(D), which also identifies the coercivity of this specific probe. Note that while $m_{x} \sim 0$, the image is clearly different from $4(\mathrm{~A})$. Weak dark strips have appeared to the left of the nominally bright areas. This suggests that the process of magnetization reversal in the $x$ direction invariably causes some moments to rotate in the opposite $z$ direction. At negative saturation, image $4(\mathrm{E})$ shows the complete reversal of the contrast, including the vertical component. Upon removal of the field, the resulting image $4(\mathrm{~F})$, which is at negative remanence, shows a somewhat weaker vertical component. It appears that some of the moments that were oriented along the $-z$ direction have reverted back to $+z$ or to the other orthogonal directions when the field was removed. The amount of $m_{z}$ retention appearing less in $4(\mathrm{~F})$ than in the positive remanence image $4(\mathrm{C})$ is due to the fact that the probe was deliberately oriented in the $+z$ direction before application of the field.

At positive coercivity, image $4(\mathrm{G})$ is analogous to $4(\mathrm{D})$, with the exception that the dark regions are affected by highfrequency noise. We speculate that since the probe is in a demagnetized state, the influence of local fields at the dark 


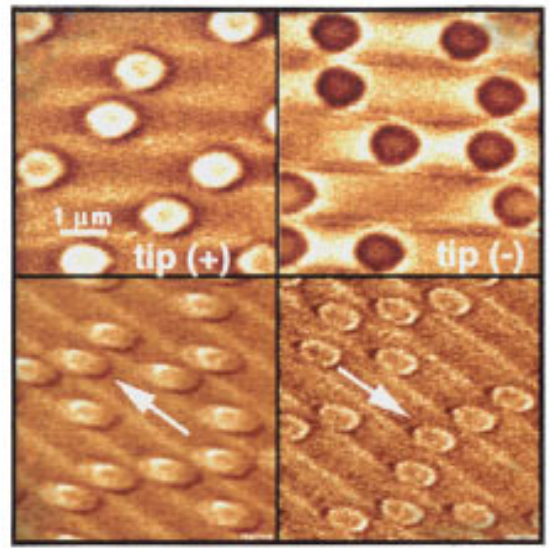

FIG. 5. MFM images of magneto-optic medium at various probe orientations. Tip $(+)$ and tip $(-)$ indicate vertically premagnetized probes, arrows indicate directions of the 600 Oe in-plane external field.

regions may induce transient switching of some magnetic moments of the probe which leads to a noisy image. As the field is brought back to positive saturation, image $4(\mathrm{H})$ appears identical to $4(\mathrm{~B})$, except that the contrast at the transition areas ( $z$ contribution) appears to be less pronounced. In successive experiments, we observed that the $z$ contribution decreases progressively and repeated cycling of the horizontal fields destroys the $m_{z}$ alignments of the probe.

In the above discussion, the probe orientation was assumed to be perpendicular to the surface. In practice, however, there is a slight angle of about $10^{\circ}$ between the main axis of the conical tip and the true vertical direction. This may account for some of the asymmetries between the equivalent points along the ascending and descending branches of the $m_{x}-H$ loop.

Probe reorientation effects for perpendicularly magnetized surfaces are a conventional magneto-optic media are shown in Fig. 5. The upper images, obtained at no bias field, correspond to a vertically oriented $m_{\text {tip }} \approx m_{z}, m_{x}, m_{y} \approx 0$ while the lower images, obtained at \pm 500 Oe horizontal applied fields, correspond to a nonvanishing $m_{x}$ component. The contrast reversals follow directly from the change in sign of Eq. (3). ${ }^{13}$ In analogy with the treatment of in-plane magnetization, we assume a simple model for the surface magnetization $M_{z}=M_{0} \sin (k x)$. The interpretation that the $z$-component imaging is sensitive to the distribution of magnetic charges still holds. One distinction, however, is that in this case the volume charge density vanishes and the discontinuity of magnetization at the surface-air interface produces the surface magnetic charges. Hence, the $z$-component images are proportional to $M_{z}$. From Laplace's equation, it follows that the $x$-component images must be proportional to the discontinuity of surface charges. In other words, the $x$-component images are sensitive to the domain walls. ${ }^{14}$ This property is illustrated in the lower images which show the bright and dark features on opposite edges of the marks which follow the direction of the applied field.

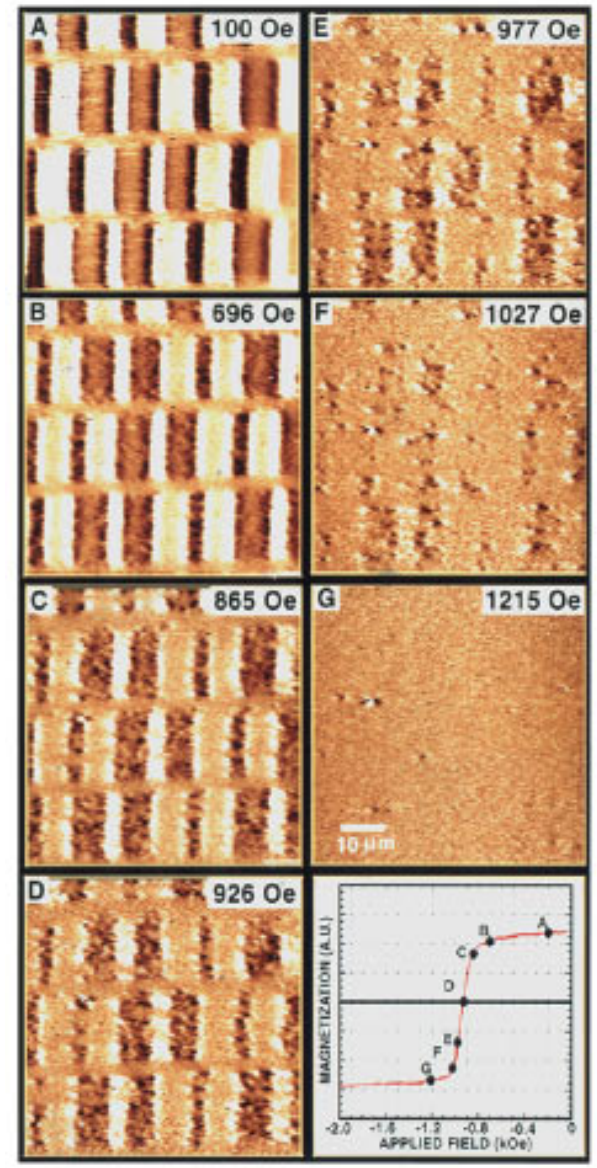

FIG. 6. Erasure of thin-film recording medium with increasing dc field.

\section{Erasure process of a thin-film medium}

Once the probe's response to the field is understood, the microscopic characteristics of the sample undergoing gradual dc erasure can be studied. A typical result is shown in Fig. 6 . The plot at the bottom is the descending branch of the measured magnetization curve, and the images are labeled according to the positions on this curve. The horizontal field reinforces the magnetization component of the pattern parallel to its direction and at the same time reverses the component in the opposite direction. Thus, the curve represents the magnetization component being reversed by the applied field.

The patterns were unperturbed by relatively weak magnetic fields, and image (A) shows the initial magnetization distribution. The system was stable up to about 700 Oe where changes in the patterns become noticeable. The initial stage occurred over some field range which is characterized by subtle expansion of the bright areas and moderate roughening of the transition regions. By taking the power spectrum of the image at $700 \mathrm{Oe}$, we obtain an average roughness correlation distance of about $0.4 \mu \mathrm{m}$, which may be related to the average switching volume for this medium. Roughening continued with increasing field and as the field approached coercivity, favorably magnetized areas conglomerated at various spots along the track. Expanding domains 


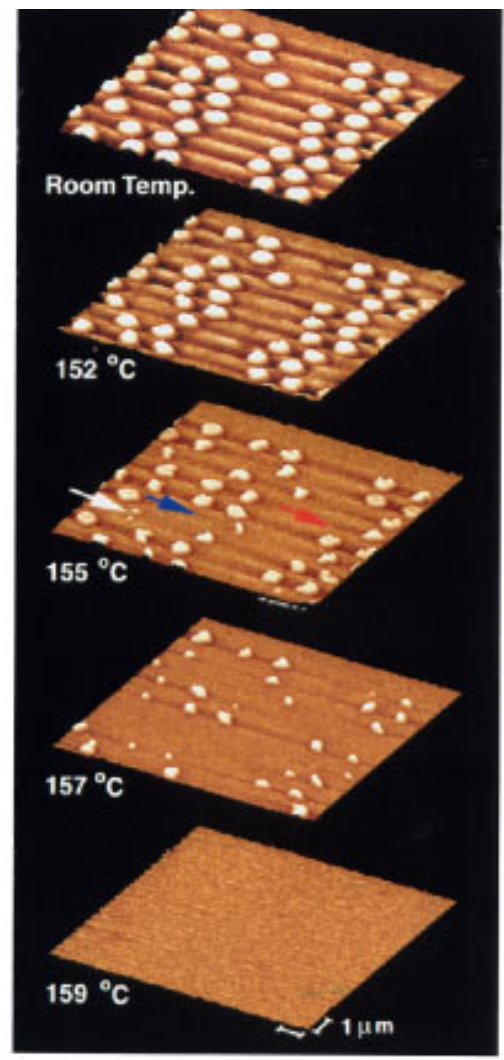

FIG. 7. Erasure of TbFeCo magneto-optic marks with increasing temperature.

adjacent to one another tended to break or percolate through the unfavorable domains. This is best observed at 865 and at 926 Oe where light-intensity streaks can be found in most of the dark contrast bits. At still higher fields, the percolated areas expanded along the crosstrack direction and at 1027 Oe, most parts have reoriented in the direction of the field. The terminal distribution of the remaining clusters appear to be uncorrelated with their previous patterns, and their random distribution may reflect the local fluctuations in the magnetic properties of the medium.

\section{Erasure process of a magneto-optic medium}

Since the coercivity of conventional magneto-optic media at room temperature is several $\mathrm{kOe}$, direct imaging of their erasure processes is inaccessible with our device. However, we show the results of a related experiment to elucidate the nature of domain collapse in these systems. These images were obtained at room temperature and under ambient magnetic field after exposure to progressively increasing temperatures in the presence of a $1.8 \mathrm{kOe}$ vertical magnetic field.

The evolution of individual magnetic marks can be seen in Fig. 7, as they were subjected to progressively increasing temperatures in a $1.8 \mathrm{kOe}$ dc external field from a permanent magnet. These images correspond to their final room- temperature configurations after recovering from the elevated temperatures. No visible perturbation of the patterns occurred with heating until about $150{ }^{\circ} \mathrm{C}$ where subtle shape variations became evident. The onset of domain movement produced irregular wall boundaries as the marks deviated from their originally circular shapes. This clearly suggests nonuniformity on a local scale and our observations are in qualitative agreement with theoretical simulations of "patchy" amorphous media with voids. ${ }^{15}$ This inhomogeneity further resulted in the multidomain character of individual marks and caused the domains to collapse at different rates. The blue and red arrows indicate the locations of marks which disappeared at $155^{\circ} \mathrm{C}$ even as most other marks were still intact. The white arrow shows direct evidence of patterns breaking up to microdomains which shrink independently. The microdomains had diameters less than $200 \mathrm{~nm}$ wide and were located on opposite grooves. The images at $157^{\circ} \mathrm{C}$ and higher exhibit the final states of domain disappearance. In general, there was no correlation between erasure characteristics and the position relative to other marks, i.e., stray magnetic fields did not influence the stability of the marks. Just prior to complete erasure, the image at $157^{\circ} \mathrm{C}$ shows that all remaining domains were those attached to the mechanical grooves, providing direct evidence that wall pinning occurred at the mechanical defects. The combination of reduced film thickness, tilted easy axis and increased substrate jaggedness at the side walls of the grooves are believed to be responsible for retarding complete domain collapse at those regions.

\section{ACKNOWLEDGMENTS}

We are grateful to A. Kratz, R. Madabhushi, M. Shih, and F. Lineberger for their assistance in various aspects of this work.

${ }^{1}$ Y. Martin and Wickramasinghe, Appl. Phys. Lett. 50, 1455 (1987).

${ }^{2}$ P. Grütter, H. J. Mamin, and D. Rugar, in Scanning Tunneling Microscopy Vol. II, edited by R. Weisendanger and H.-J. Guntherodt (Springer, Berlin, 1992), p. 151.

${ }^{3}$ U. Hartmann, J. Vac. Sci. Technol. A 8, 411 (1990).

${ }^{4}$ R. Proksch, T. E. Schäffer, B. M. Moskowitz, E. D. Dahlberg, D. A. Bazylinski, and R. B. Frankel, Appl. Phys. Lett. 66, 2582 (1995).

${ }^{5}$ R. D. Gomez, I. D. Mayergoyz, and E. R. Burke, IEEE Trans. Magn. 31, 3346 (1995).

${ }^{6}$ T. Albrecht, P. Grütter, D. Horne, and D. Rugar, J. Appl. Phys. 69, 668 (1991).

${ }^{7}$ Nanoscope III Multimode SPM, Digital Inst. Inc., Cortuna, CA.

${ }^{8}$ U. Dürig, O. Züger, and A. Stalder, J. Appl. Phys. 72, 1778 (1992).

${ }^{9}$ R. D. Gomez, A. R. Kratz, E. R. Burke, and I. D. Mayergoyz, IEEE Trans. Magn. 31, 3265 (1995).

${ }^{10} \mathrm{R}$. L. Wallace, in Introduction to Magnetic Recording, edited by R. L. White (IEEE, New York, 1985), p. 226.

${ }^{11}$ D. Rugar, H. J. Mamin, P. Guethner, S. E. Lambert, J. E. Stern, I. McFadyen, and T. Yogi, J. Appl. Phys. 68, 1179 (1990).

${ }^{12}$ R. D. Gomez, I. D. Mayergoyz, and E. R. Burke, J. Appl. Phys. 75, 5910 (1994).

${ }^{13}$ S. Foss, R. Proksch, and E. D. Dahlberg, J. Magn. Soc. Jpn. 19, S1, 135 (1995).

${ }^{14}$ D. Rugar, H. J. Mamin, R. Erlandsson, J. E. Stern, and B. D. Terris, Rev. Sci. Instrum. 59, 2339 (1988).

${ }^{15}$ M. Mansuripur, R. Giles, and G. Patterson, J. Appl. Phys. 69, 4844 (1991). 
Journal of Applied Physics is copyrighted by the American Institute of Physics (AIP). Redistribution of journal material is subject to the AIP online journal license and/or AIP copyright. For more information, see http://ojps.aip.org/japo/japcr/jsp

Copyright of Journal of Applied Physics is the property of American Institute of Physics and its content may not be copied or emailed to multiple sites or posted to a listserv without the copyright holder's express written permission. However, users may print, download, or email articles for individual use. 